\title{
Real-Time 100-GS/s Sigma-Delta All-Digital Radio-over- Fiber Transmission for 22.75-27.5 GHz Band
}

\author{
H. Li ${ }^{1}$, M. Verplaetse ${ }^{1}$, J. Verbist ${ }^{1,2}$, J. Van Kerrebrouck ${ }^{1}$, L. Breyne ${ }^{1,2}$, C.-Y. Wu ${ }^{1}$, \\ L. Bogaert ${ }^{1,2}$, X. Yin, J. Bauwelinck ${ }^{1}$, P. Demeester ${ }^{1}$ and G. Torfs ${ }^{1}$ \\ ${ }^{1}$ IDLab, INTEC, Ghent University - imec, 9052 Ghent, Belgium \\ ${ }^{2}$ Photonics Research Group, INTEC, Ghent University - imec, 9052 Ghent, Belgium \\ haolin.li@ugent.be
}

\begin{abstract}
We present the first real-time 100-GS/s FPGA-based sigma-delta modulator to enable all-digital radio-over-fiber transmission in the $22.75-27.5 \mathrm{GHz}$ band. $4.68 \mathrm{~Gb} / \mathrm{s}(2.34 \mathrm{~Gb} / \mathrm{s}) 64-\mathrm{QAM}$ is transported over $10-\mathrm{km} \mathrm{SSMF}$ at $1562 \mathrm{~nm}$ with $6.46 \%$ (4.73\%) EVM.
\end{abstract}

\section{Introduction}

The fifth generation wireless networks $(5 \mathrm{G})$ drives research in the direction of massive device connectivity, high data rates and decreased latency. One of the prominent features of $5 \mathrm{G}$ is the deployment of small cells, creating a large quantity of remote radio heads (RRHs) [1]. Cloud radio access networks (C-RANs) in combination with radio-overfiber (RoF) can be a key-enabling technology to realize this [2]. Three different realizations of the radio-over-fiber link, including digitized radio-over-fiber (DRoF), analog radio-over-fiber (ARoF) and sigma-delta-over-fiber (SDoF), have been discussed in our previous works $[3,4]$. SDoF simplifies RRHs by oversampling the signal and translating it to a bi-level signal, leveraging the benefits of both the DRoF (allowing low-cost telecom components) and ARoF (low-complexity RRH). Recently, we have verified the performance of this SDoF approach for sub-6 GHz systems by transmitting 4 parallel lanes of $3.5 \mathrm{~Gb} / \mathrm{s} 256-\mathrm{QAM}$ signals on a $3.5 \mathrm{GHz}$ carrier over $20 \mathrm{~km}$ standard single-mode fiber (SSMF) at $1310 \mathrm{~nm}$ [4]. However, moving to higher frequency bands such as $>24 \mathrm{GHz}$ bands [5], SDoF has not been reported owing to the limited sampling rate of the state-of-the-art sigma delta modulators (SDMs) [6]. Therefore, when a simple RRH configuration is required, prior works mainly rely on the ARoF, where the baseband or IF signal is translated to the carrier frequency by either analog or optical up-conversion [7]. However, the main drawbacks of this approach are the sensitivity to nonlinearities in the E/O conversion and the requirement of additional components for frequency up-conversion.

Our recent work eliminates the sampling rate limitation of SDMs and shifts high-frequency operations to a singlebit multiplexer [6], making the realization of a real-time SDoF transmission for $>24 \mathrm{GHz}$ band technically feasible. By combining the SDM parallelization technique for FPGAs with an in-house developed high-speed multiplexer [8], we have successfully extended the SDM's sampling rate from $21 \mathrm{GS} / \mathrm{s}$ in [6] to $100 \mathrm{GS} / \mathrm{s}$ ( $\approx 5 \mathrm{x}$ increase), which is the fastest SDM ever reported. As an upgrade of sub- $6 \mathrm{GHz}$ SDoF systems, we present the first demonstration of a realtime 100-GS/s all-digital SDoF transmission at $1562 \mathrm{~nm}$ covering the $22.75-27.5 \mathrm{GHz}$ band in the $5 \mathrm{G}$ context [5].

\section{Experimental Setup}

The experimental setup and its block diagram are illustrated in Fig. 1. A Xilinx Virtex Ultrascale FPGA VCU108 was adopted to generate the sigma-delta signals. On FPGA, a baseband (BB) or low-IF QAM-signal (roll-off 0.28, 390.625 MBd per channel, FPGA clock frequency of $390.625 \mathrm{MHz}$ ) is first oversampled and noise-shaped by lowpass secondorder SDMs at an equivalent sampling rate of $50 \mathrm{GS} / \mathrm{s}$ for both I and Q channels using the parallelization techniques proposed in [6]. The parallel SDMs use a parallelization degree of 128 and have a latency of approx. 270 samples or

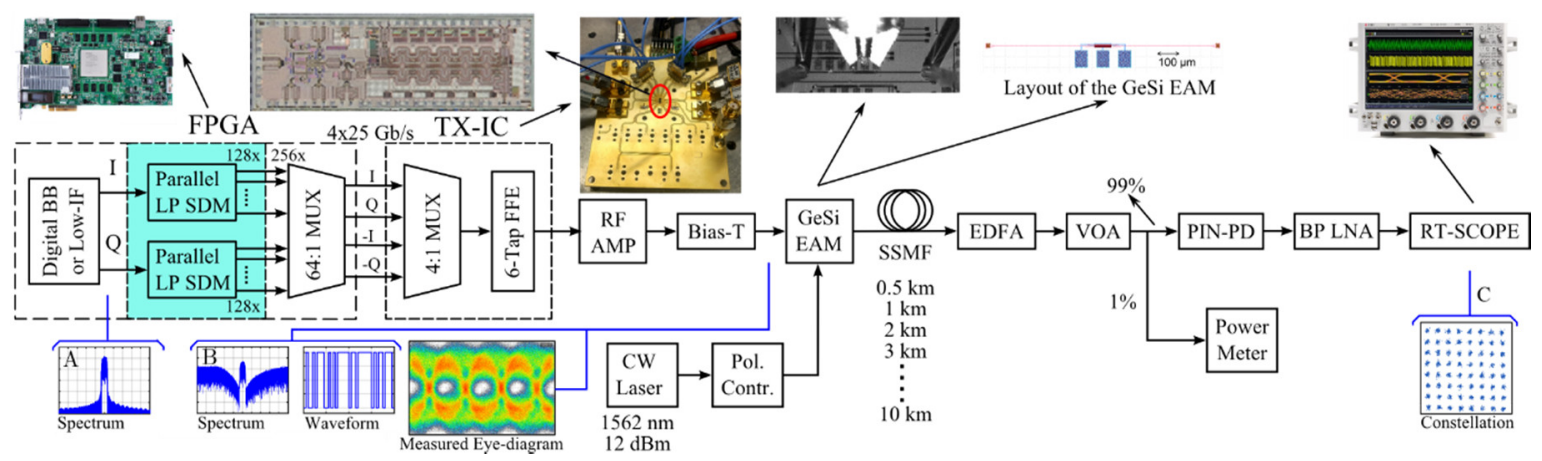

Fig. 1. Block diagram and experimental setup for the real-time 100-GS/s sigma-delta radio-over-fiber transmission. 

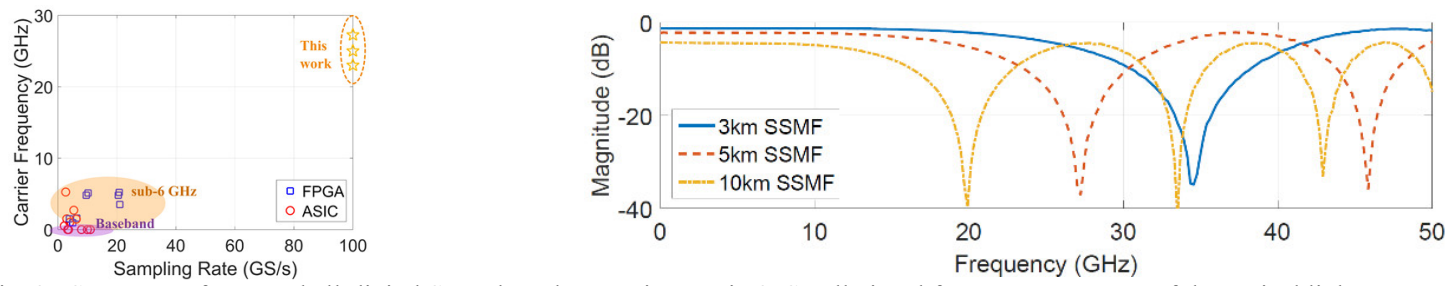

Fig. 2. Summary of reported all-digital SDM-based transmitters. Fig.3. Small-signal frequency response of the optical link, comprising a GeSi EAM and a $50 \mathrm{GHz}$ commercial PIN-PD.

$691 \mathrm{~ns}$. The low-IF signal is generated using a polyphase digital direct synthesis (DDS) module. The 256 parallel streams are interleaved into 4 streams at $25 \mathrm{~Gb} / \mathrm{s}$ to drive four FPGA high-speed serial interfaces. These interfaces generate I, Q, -I, -Q samples that are used for the subsequent up-conversion to $25 \mathrm{GHz}$.

An in-house developed transmitter IC (TX-IC) multiplexes these $4 \times 25 \mathrm{~Gb} / \mathrm{s}$ streams into a serial $100 \mathrm{~Gb} / \mathrm{s}$ singlebit stream, accomplishing the digital up-conversion to a $25 \mathrm{GHz}$ carrier. Note the second-order lowpass SDMs and the digital up-conversion result in an equivalent $100 \mathrm{GS} / \mathrm{s}$ fourth-order bandpass SDM with a $25 \mathrm{GHz}$ carrier frequency. This is the fastest SDM ever reported and is also the first SDM for $>24 \mathrm{GHz}$ band without analog/optical up-conversion. Fig. 2 shows a summary of reported all-digital SDM-based transmitters for BB or RF signals, implemented by ASIC or FPGA. The utilized TX-IC is fabricated in a $55 \mathrm{~nm}$ SiGe BiCMOS technology and consumes $0.85 \mathrm{~W}$. In addition to a multiplexer, the TX-IC also contains a 6-tap feedforward equalizer (FFE), which was tuned to compensate the bandwidth limitation of the interconnect towards a silicon-based electroabsorption modulator (EAM) [8]. To boost the generated output signal of approximately $300 \mathrm{mVpp}$ single-ended signals, two $67 \mathrm{GHz}$ broadband RF-amplifiers amplify the signal from the TX-IC via an RF-probe to the bondpads of the EAM.

The EAM for the E/O conversion is a very compact GeSi EAM $(<100 \mu \mathrm{m}$, see inset Fig. 1 with $>50 \mathrm{GHz}$ bandwidth fabricated on a silicon photonics platform similar to the one in reference [8]. Light is coupled in and out of the waveguide structure through fiber-to-chip grating couplers with an insertion loss of $\sim 6 \mathrm{~dB}$ per coupler. Owing to the digital up-conversion, neither analog nor optical up-conversion is necessary and the EAM is directly driven by bilevel signals, resulting in a simple transmitter structure.

At the receiver/RRH, an erbium-doped optical amplifier (EDFA) and a variable optical attenuator (VOA) were used to control the signal power before a commercial $50 \mathrm{GHz}$ III-V-based $50 \Omega$ PIN-PD. A narrowband receiver (realized by a bandpass low noise amplifier (LNA) in this work) is preferable as it can help remove the out-of-band quantization noise shaped by the SDMs. The PD and LNA only need to receive the signal of interest around $25 \mathrm{GHz}$ carrier, it is not necessary to receive the $100 \mathrm{~Gb} / \mathrm{s} \mathrm{NRZ/OOK} \mathrm{signal.} \mathrm{A} \mathrm{Keysight} \mathrm{real-time} \mathrm{oscilloscope} \mathrm{(DSAZ634A)}$ and VSA software were used to demodulate QAM-signals.

\section{Results and Discussion}

The fiber channel plays a critical role in the C-band link, where the relatively large chromatic dispersion coefficient manifests itself as notches in the frequency response. The small-signal frequency response of a link consisting of the EAM (-0.75 V bias) and the PIN-PD is given in Fig. 3 for different fiber spans $(3 \mathrm{~km}, 5 \mathrm{~km}$ and $10 \mathrm{~km})$ at $1562 \mathrm{~nm}$.

The measured spectra at the RF amplifier output in electrical back-to-back (B2B) and at the $50 \mathrm{GHz}$ PIN-PD output in optical B2B are shown in Fig. 4a where signal-to-noise ratios (SNRs) of $32.2 \mathrm{~dB}$ and $29.1 \mathrm{~dB}$ are indicated as a performance reference. The low in-band quantization noise (from $24 \mathrm{GHz}$ to $26 \mathrm{GHz}$ ) allows us to transmit two channels $(\mathrm{CH} 1$ at $24.7 \mathrm{GHz}$ and $\mathrm{CH} 2$ at $25.3 \mathrm{GHz}$ ) simultaneously. Unless stated otherwise, the following measurements are referred to single channel measurements at $25 \mathrm{GHz}$.

The signal quality was investigated as a function of the optical input power on the PIN-PD in Fig. 4b. The signal quality improves with higher optical input powers and the root-mean-square (rms) value of the error vector magnitude (EVM) drops below 5\%, enabling adequate signal quality for 64-QAM with 1E-05 bit error rates (BERs) without error coding. The total power consumption of this SDoF link is low by avoiding components such as high-speed DACs and frequency up-converters, and can be further reduced by using a low-power EAM NRZ driver with continuous-time equalization [9] as this driver removes the necessity for the FFE and RF amplifiers in Fig.1.

To explore the reach of this SDoF transmission, the EVMs were compared among different fiber spans in Fig. 5a. The measured EVMs are 3.09\% and 3.76\% for electrical B2B and optical B2B (at $8 \mathrm{dBm}$ optical received power) in single channel measurements, respectively. The EVMs slightly increases to $4.2 \%$ and $5.43 \%$ in the two channel measurements, respectively. No noticeable degradation in EVM has been observed for fiber spans below $3 \mathrm{~km}$. The high EVMs ( $>25 \%)$ at $6 \mathrm{~km}$ fiber are caused by the notch around $25 \mathrm{GHz}$ due to chromatic dispersion of the SSMF for $1562 \mathrm{~nm}$, which is in good agreement with the S-parameter measurements in Fig. 3. The peak EVMs between CH1 and $\mathrm{CH} 2$ are slightly shifted owing to different carrier frequencies. For $10 \mathrm{~km}$ distance, the notch is shifted away from 
the carrier frequency, leading again to a good EVM. This EVM degradation caused by the chromatic dispersion notch can be alleviated by adding a dispersion compensation module (DCM). Only a small degradation in EVM is observed from optical B2B to $10 \mathrm{~km}$ fiber due to optical insertion loss. The demodulated constellations of the 64-QAM signals are compared in Fig. $5 \mathrm{~b}$ for single channel and two channel cases. These EVM values are consistent with the measured SNR in Fig. 4a. The total bit rate of the 64-QAM signal is approximately $2.34 \mathrm{~Gb} / \mathrm{s}$ in the single channel case and is doubled in the two channels case. Moreover, by digitally shifting the IF frequency with DDS, this all-digital transmitter covers a wide frequency band from $22.75 \mathrm{GHz}$ to $27.5 \mathrm{GHz}$ (with $500 \mathrm{MHz}$ bandwidth around carrier frequencies 23$27.25 \mathrm{GHz}$ ) with relatively low EVMs as depicted in Fig. 5c. It shows the superior EVMs in the single channel case (electrical B2B) e.g. EVM of $3.58 \%$ compared to $4.2 \%$ in two channel case at $24.7 \mathrm{GHz}$ carrier. This frequency range covers the 5G NR band n258 specified in 3GPP [5] and these EVMs satisfy the 3GPP requirement for 64-QAM: EVM $<8 \%[10]$.

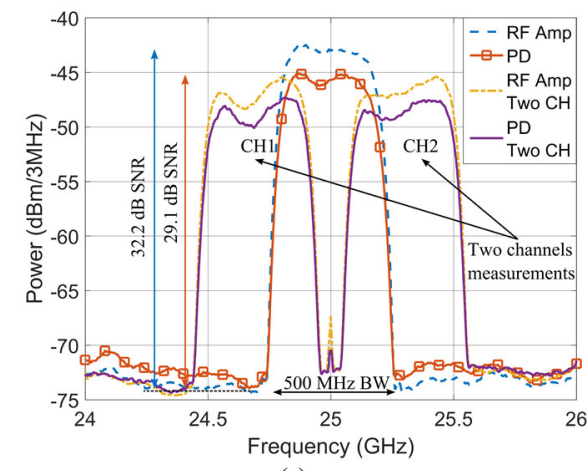

(a)

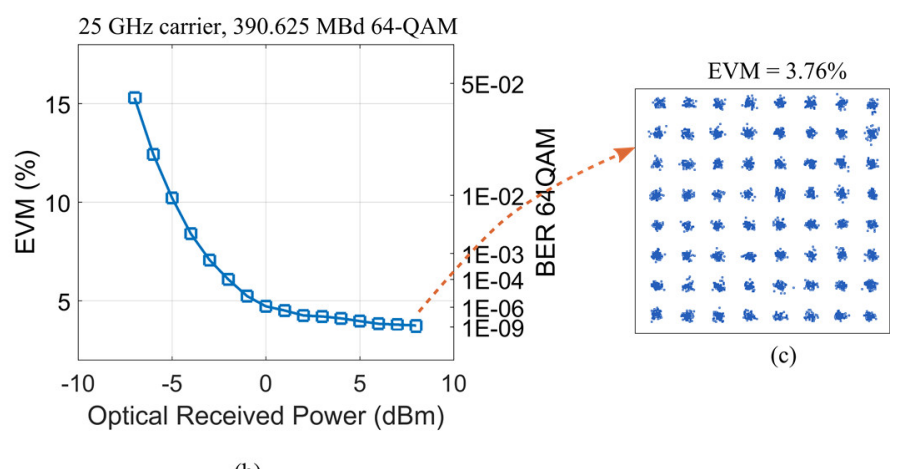

(b)

Fig. 4. (a) Measured in-band spectra at RF amplifier and PIN-PD outputs. (b) Measured rms EVM as a function of the optical power received by the PD in the single channel case (optical B2B). (c) Demodulated constellation.

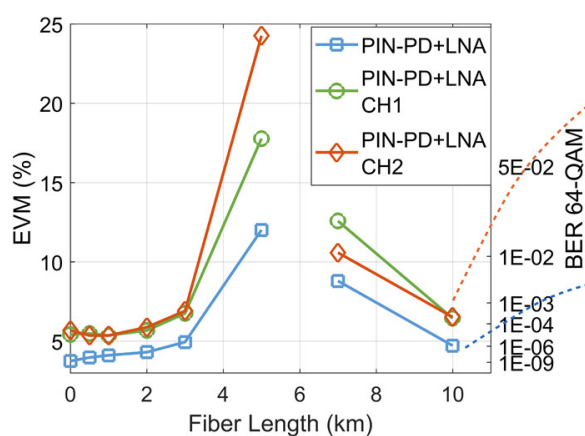

(a)

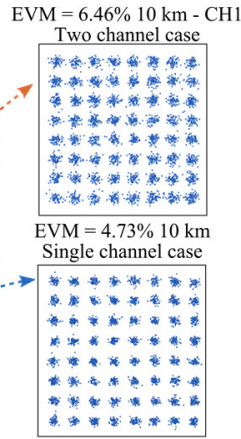

(b)

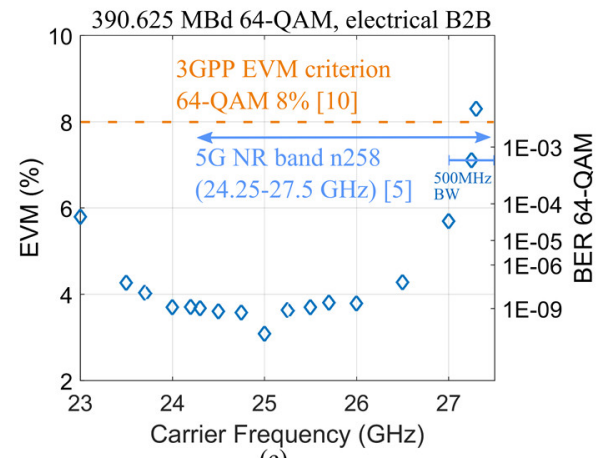

(c)

Fig. 5. (a) Measured rms EVM vs fiber spans. (b) Demodulated constellation. (c) Measured rms EVM vs carrier frequency in electrical B2B.

\section{Conclusion}

We have demonstrated the first real-time sigma-delta all-digital radio-over-fiber transmission in the $22.75-27.5 \mathrm{GHz}$ band without the aid of analog/optical up-conversion enabled by a fast 100-GS/s sigma-delta modulator. The simple structure and low latency of the transmitter and RRH, digital reconfigurability of carrier frequency, and the prominent performance (4.68 Gb/s 64-QAM at low EVMs) corroborate the strong competitiveness of the SDoF approach in highfrequency band radio-over-fiber $5 \mathrm{G}$ communications.

\section{References}

[1] G. Torfs et al., "ATTO: wireless networking at fiber speed," JLT, vol. 36, no. 8, pp. 1468-1477, Apr. 2018.

[2] C. Ranaweera et al., "5G C-RAN with optical fronthaul: an analysis from a deployment perspective," JLT, vol. 36, no. 11, Jun. 2018.

[3] L. Breyne et al., "Comparison between analog radio-over-fiber and sigma delta modulated radio-over-fiber," PTL, vol. 29 , no. 21 , Nov. 2017.

[4] C.-Y. Wu et al., "Real-time 4x3.5 Gbps sigma delta radio-over-fiber for a low-cost 5G C-RAN downlink," in Proc. of ECOC, 2018.

[5] "3GPP Release 15 Overview," [Online]. Available: https://spectrum.iee.org/telecom/wireless/3gpp-release-15-overview.

[6] H. Li et al., "A 21-GS/s single-bit second-order delta sigma modulator for FPGAs," TCAS-II (2018) [Early Access].

[7] K.V. Gasse et al., "Analog radio-over-fiber transceivers based on III-V-on-silicon photonics," PTL (2018) [Early Access].

[8] J. Verbist et al., "Real-time $100 \mathrm{~Gb} / \mathrm{s}$ NRZ and EDB transmission with a GeSi electroabsorption modulator for short-reach optical interconnects," JLT, vol. 36, no. 1, pp. 90-96, Jan. 2018.

[9] H. Ramon et al., "70 Gb/s 0.87 pJ/bit GeSi EAM driver in 55nm SiGe BiCMOS," in Proc. of ECOC, 2018.

[10] 3GPP TS 36.104: "Evolved Universal Terrestrial Radio Access (E-UTRA): Base Station (BS) radio transmission and reception," V15.2.0, 2018-03. (Release 15). 